\title{
RELATION BETWEEN HARDNESS OF (Ti, Al)N BASED MULTILAYERED COATINGS AND PERIODS OF THEIR STACKING
}

\author{
Mehdi Zaoui ${ }^{a, b}$, Alexandre Bourceret ${ }^{a}$, Yves Gaillard ${ }^{a}$, \\ Sylvain Giljean ${ }^{c, *}$, Christophe Rousselot ${ }^{a}$, Marie-José PaC ${ }^{c}$, \\ FABRice RICHARD ${ }^{a}$ \\ ${ }^{a}$ Université Bourgogne Franche-Comté, Institut FEMTO-ST, CNRS/UFC/ENSMM/UTBM, 32 avenue de \\ l'Observatoire, 25000 Besançon, France \\ ${ }^{b}$ Institut de recherche technologique, Matériaux, Métallurgie, Procédés, 4 Rue Augustin Fresnel, 57070 METZ, \\ France \\ ${ }^{c}$ Université de Haute Alsace, LPMT, 2 Rue des Frères Lumière, 68093 Mulhouse, France \\ * corresponding author: sylvain.giljean@uha.fr
}

ABstract. This study aims to model, by using a finite element method, the relationship between the hardness and the period $\Lambda$ of metal/nitride multilayer coatings $\left(\mathrm{Ti}_{0.54} \mathrm{Al}_{0.46} / \mathrm{Ti}_{0.54} \mathrm{Al}_{0.46} \mathrm{~N}\right)_{\mathrm{n}}$ in order to understand the increase of the hardness at the low periods [1] and then optimise the multilayer coating architecture to obtain the best mechanical properties. A 2D axisymmetric finite element model of the Berkovich nanoindentation test was developed. The coating was designed as a stacking of $\operatorname{Ti}_{0.54} \mathrm{Al}_{0.46}$ and $\mathrm{Ti}_{0.54} \mathrm{Al}_{0.46} \mathrm{~N}$ nanolayers with, in the first hypothesis, equal thickness and perfect interface. The elastoplastic behaviours of the metal and nitride layers were identified by Berkovich nanoindentation experiments and inverse analysis on thick monolayer samples. The indentation curves $(\mathrm{P}-\mathrm{h})$ obtained by this model depend on the period $\Lambda$ of the stacking. Simulated (P-h) curves were compared with experimental data on $2 \mu \mathrm{m}$ thick films with different periods $\Lambda$ ranging from 10 to $50 \mathrm{~nm}$ deposited by $\mathrm{RF}$ magnetron sputtering using reactive gas pulsing process (RGPP). The model forecasts are very consistent with the experience for the largest period but the model does not reproduce the hardness increase at the lowest periods. The $\Lambda=10 \mathrm{~nm}$ coating was analysed by electron energy loss spectroscopy (EELS) on a transmission electron microscope. Results show intermixing of the layers with the presence of nitrogen atoms in the metal layer over a few nanometers [1. It was concluded that the metal/ceramic interface plays an important role at low periods. The addition in the model of a transition layer in the metal/nitride stacking, with an elastoplastic metal/ceramic medium behaviour, allows to reproduce the nanoindentation experimental curves. The thickness of this transition layer deduced from model updating method is in very good agreement with EELS observations.

KEYwords: Finite element method, hardness, indentation modulus, nanoindentation, TiAl/TiAlN multilayer films.

\section{INTRODUCTION}

Titanium and aluminium nitrides (TiAl) N coatings are extensively used in the industry since many years for their hardness, toughness and wear and oxidation resistances [2, 3]. It has been shown that the indentation modulus and the hardness of $\mathrm{Ti}_{1-\mathrm{x}} \mathrm{Al}_{\mathrm{x}} \mathrm{N}$ coatings depend on the aluminium content and that the optimal properties are obtained when the maximum $\mathrm{Al}$ content is substituted for $\mathrm{Ti}$ in face centred cubic structure close to $\mathrm{x}=0.46[1,4]$. One way to improve the hardness of that kind of coatings consists in depositing multilayer films.

In this study, multilayer coatings are studied and consist in metal/ceramic stacking with alternate deposition of $\mathrm{Ti}_{0.54} \mathrm{Al}_{0.46}$ and $\mathrm{Ti}_{0.54} \mathrm{Al}_{0.46} \mathrm{~N}$ nanolayers with three different periods of the bilayer metal/ceramic $\Lambda=50,16,10 \mathrm{~nm}$. In order to facilitate the reading of the paper, $\mathrm{Ti}_{0.54} \mathrm{Al}_{0.46}$ and
$\mathrm{Ti}_{0.54} \mathrm{Al}_{0.46} \mathrm{~N}$ are respectively written as $\mathrm{TiAl}$ and TiAlN. The main objective of the paper is to study the relationship between hardness of as-design multilayer films and periods of their stacking to understand the elastoplastic phenomena that are involved in the hardness increase experimentally observed at the lowest periods [1].

A 2D axisymmetric model of the Berkovich nanoindentation is developed. Then a method to identify the elastoplastic mechanical properties of the monolayer TiAl and TiAlN is proposed. The results are introduced in a multilayer stacking model that allows to obtain the indentation curves (P-h) as a function of the period $\Lambda$. As a first hypothesis the interface between TiAl and TiAlN layers is considered as perfect without thickness. Then a transition layer is added and allows to reproduce the experimental indentation curves. 


\section{Materials AND Methods}

TiAl and TiAlN monolayer thin films were deposited onto $\mathrm{Si}$ (100) wafer by RF magnetron sputtering using one sintered titanium/aluminium 66/33 at.\% alloy target as described elsewhere [4. Same experimental conditions were used to deposit (TiAl/TiAlN)n multilayer films with different periods using Reactive Gas Pulsing Process (RGPP) [5] with a controlled pulsing flow rate of the nitrogen reactive gas as described elsewhere [1]. Finally, the samples considered in this paper are summarised in Table 1

\begin{tabular}{ccc}
\hline Sample & $\begin{array}{c}\text { Period } \\
\Lambda(\mathrm{nm})\end{array}$ & $\begin{array}{c}\text { Thickness } \\
\text { ep }(\mu \mathrm{m})\end{array}$ \\
\hline TiAl & $/$ & 3.0 \\
TiAlN & $/$ & 3.0 \\
& 50 & 2.0 \\
$(\text { TiAl/TiAlN })_{\mathrm{n}}$ & 16 & 2.0 \\
& 10 & 1.8 \\
\hline
\end{tabular}

TABLE 1. Sample characteristics.

Nano-indentation tests were performed using an Ultra Nano-indentation Hardness Tester provided by Anton Paar. Experiments were conducted using a Berkovich tip and the multicycle procedure in load control up to $8 \mathrm{mN}$ [1, 4].

A 2D axisymmetric finite element model of the Berkovich nanoindentation test was developed with the ANSYS finite element software as described on Figure 1. An implicit method with non-linear geometric formulation has been used with higher order $2 \mathrm{D}$ elements. A deformable indenter, with isotropic linear elastic law, was modelled with a half contact angle of $70.3^{\circ}$ and a tip radius to fit the experimental tip defects. A friction coefficient of 0.1 was imposed at the contact between the indenter and the film. The mesh density has been refined so that the smallest elements are localised in the contact region. The film can be either monolayer TiAl or TiAlN or multilayer $(\mathrm{TiAl} / \mathrm{TiAlN})_{\mathrm{n}}$. For the monolayer TiAl and TiAlN, isotropic elastoplastic behaviour laws were considered with linear hardening. Elastic properties were obtained from experimental nanoindentation and plastic properties were estimated using finite element model updating.

The approach used to extract the constitutive parameters of plastic law of TiAl and TiAlN consists in an optimisation problem; the objective of which is to minimise a cost function $\omega$ by using an algorithm based on the Levenberg-Marquardt [6] 7] method using the MIC2M software [8]:

$$
\widehat{\theta}=\underset{\theta}{\operatorname{argmin}} \omega\left(P(t ; \theta), P^{e x p}(t)\right)
$$

where $\widehat{\theta}$ is the vector of the unknown parameters and $\omega$ the cost function i.e., the gap between $P^{\exp }(t)$ the

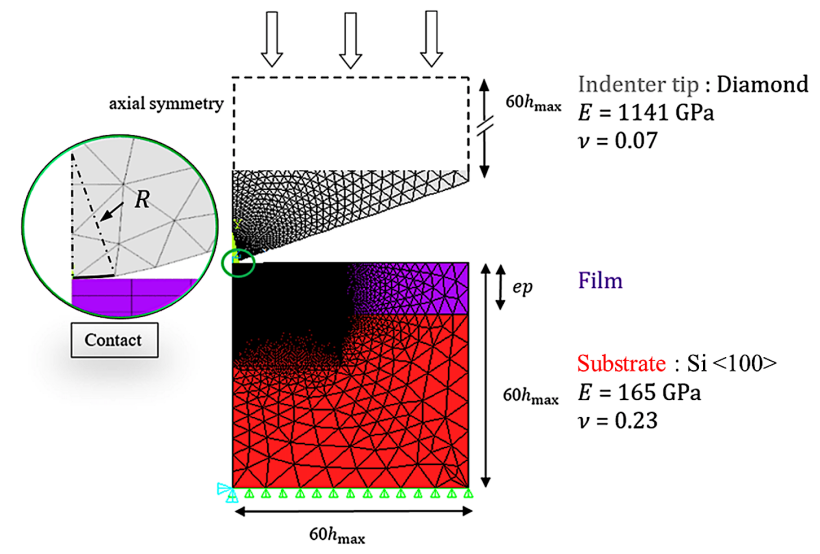

Figure 1. Description of the 2D axisymmetric finite element indentation model.

experimental data and $P(t ; \theta)$ the numerical results. Then the cost function can be defined as:

$$
\omega(\theta)=\frac{1}{2 T} \sum_{k=1}^{T}\left(\frac{P_{k}(\theta)-P_{k}^{e x p}}{P_{\max }^{e x p}}\right)^{2}
$$

where $T$ is the number of acquisition times of the load $P_{k}(\theta)=P\left(t_{k} ; \theta\right)$ and $P_{\max }^{e x p}$ the maximum value of the experimental indentation load.

\section{Results AND Discussion}

\subsection{Mechanical properties of TiAl and TIALN MONOLAYERS}

All the experimental data of the indentation tests performed on the TiAl and TiAlN monolayer films have already been published elsewhere 1, 4. Hardness and indentation modulus values of the metal TiAl are significantly lower than the ones of nitride TiAlN. By postulating the Poisson's ratio as known, the elastic properties of the monolayer films can be deduced from the experimental data. Then, plastic parameters were numerically deduced from the optimisation approach described in method section. Results are presented in Table 2. The comparison of the experimental and model indentation load curves are plotted in Figure 2 and show a good agreement.

\subsection{Simulation of $(\text { TiAl/TiAlN })_{\mathrm{N}}$ MULTILAYER FILMS WITH PERFECT INTERFACES}

All the experimental data of the indentation tests performed on the multilayer $(\mathrm{TiAl} / \mathrm{TiAlN})_{\mathrm{n}}$ films have already been published elsewhere [1. Interesting observation is that both the hardness and the elastic modulus of the multilayer films follow a Hall and Petch law as the values linearly increase as a function of $1 / \sqrt{\Lambda}$. In a first approach, the multilayer film was modelled as a stacking of nanolayers of TiAl and TiAlN with a perfect interface without thickness as showed in Figure 3 a. The model was set for each 


\begin{tabular}{ccccc}
\hline & $\begin{array}{c}\text { Young Modulus } \\
(\mathrm{GPa})\end{array}$ & Poisson ratio & $\begin{array}{c}\text { Yield stress } \\
(\mathrm{GPa})\end{array}$ & $\begin{array}{c}\text { Linear hardening } \\
(\mathrm{GPa})\end{array}$ \\
\hline TiAl & 199 & 0.25 & 0.88 & 56.6 \\
TiAlN & 417 & 0.21 & 5.70 & 405.0 \\
\hline
\end{tabular}

TABLE 2. Elastoplastic parameters of the metal TiAl and the nitride TiAlN.
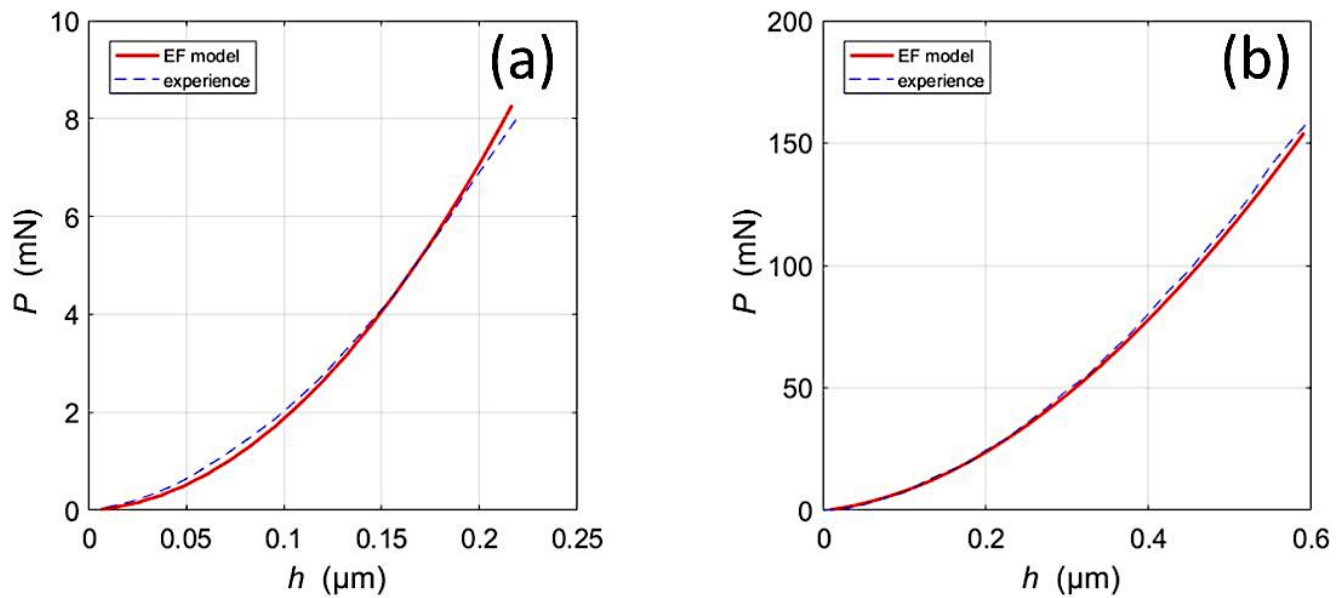

Figure 2. Loading indentation curves (P-h) of (a) TiAl and (b) TiAlN monolayers.

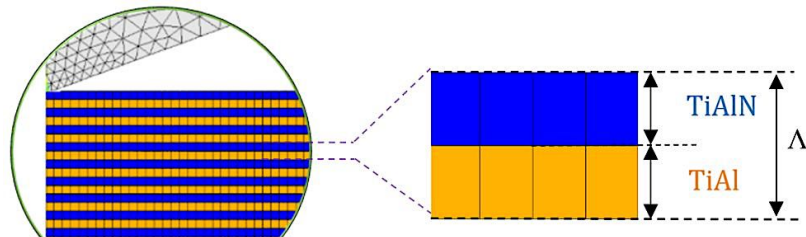

(a)

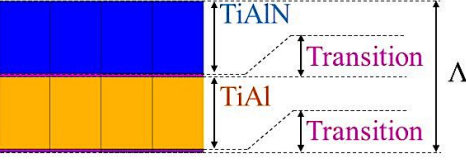

(b)

FiguRE 3. Modelling of multilayer films, (a) with perfect interfaces (b) with a transition layer.
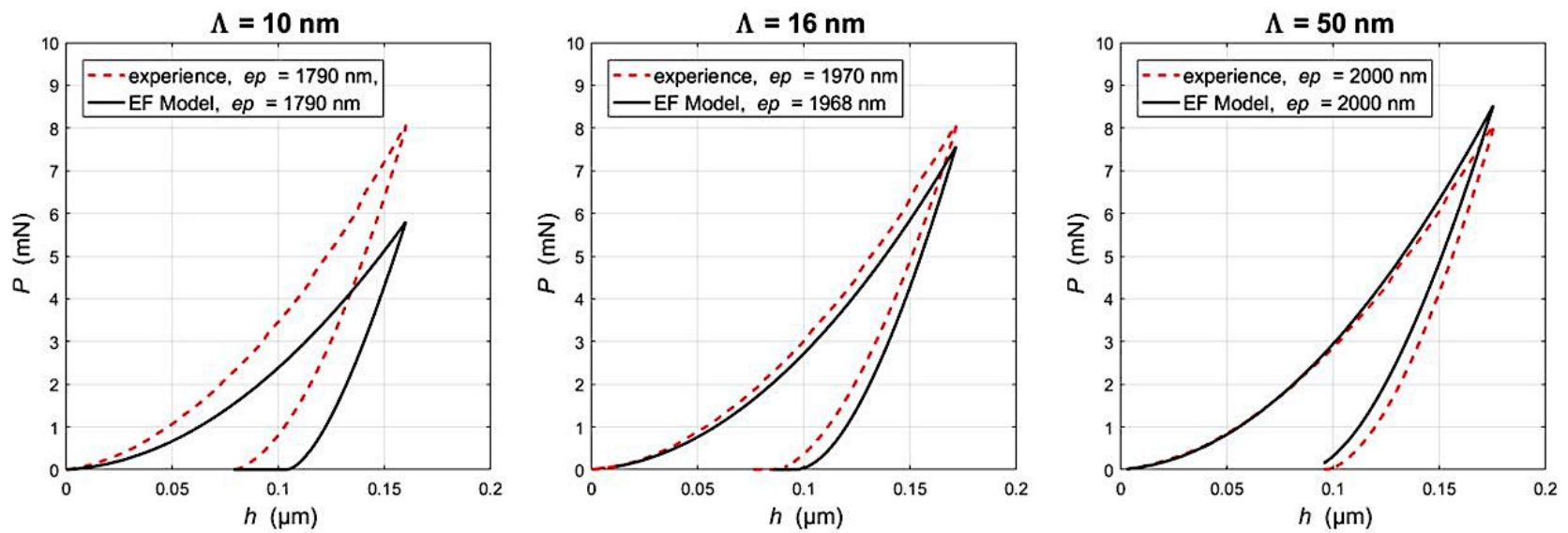

Figure 4. Comparison of the (P-h) experimental and simulated curves for $\Lambda=10,16$ and $50 \mathrm{~nm}$ with a perfect interface between TiAl and TiAlN nanolayers.

stacking period $\Lambda=10,16$ and $50 \mathrm{~nm}$. A convergence study was carried out with 1, 2 or 3 elements in the thickness of the nanolayers. Since the relative variation of the load is less than $0.5 \%$, only one element, with a $\Lambda / 2$ size, was finally used in the thickness (Figure $3 \mathrm{a}$ ), in order to minimise the calculation time. Results of the as simulated (P-h) curves are plotted on Figure 4. For the largest period $\Lambda=50 \mathrm{~nm}$, the model is in good agreement with the experience. Furthermore, the gap between the experimental curves and the simulated ones increases when the period decreases to become important for the smallest period. Therefore, the phenomenon that generates an increase in hardness when period decreases is not taken into 

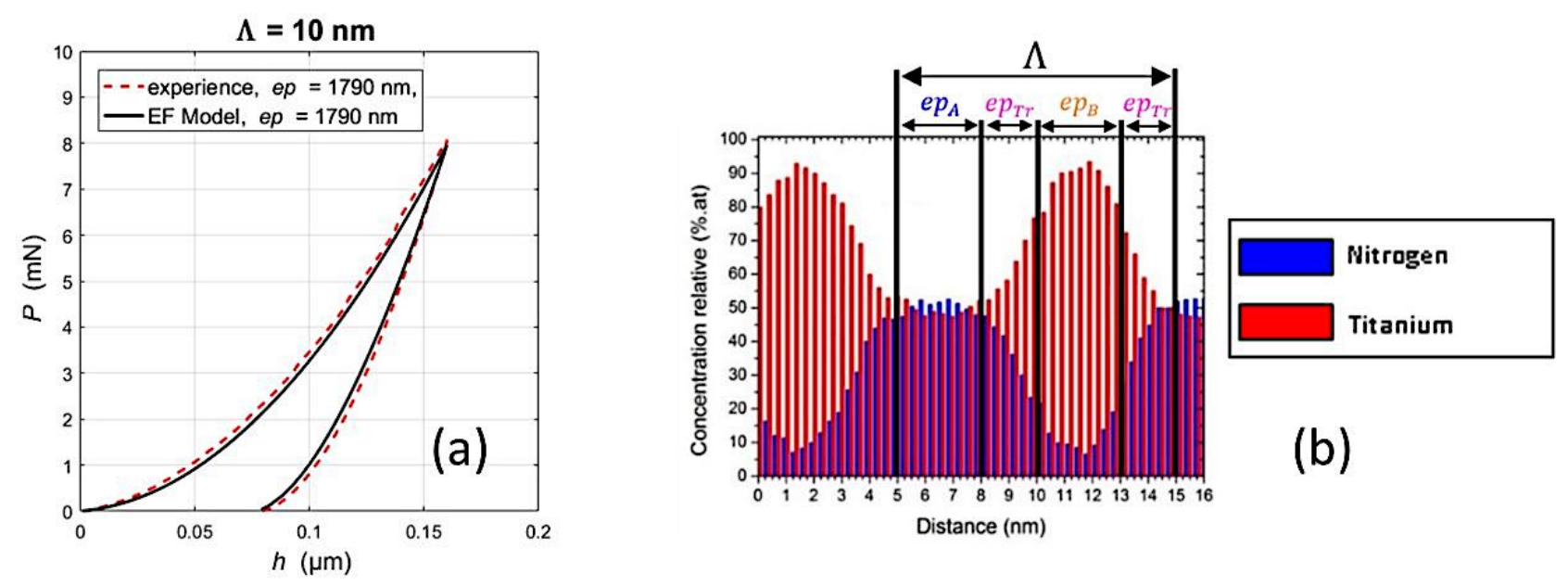

Figure 5. For $\Lambda=10 \mathrm{~nm}$, (a) Comparison of the (P-h) experimental and simulated curves with a transition layer, (b) EELS results.

account in the coating model without interfaces.

\subsection{Simulation of $(\text { TiAl/TiAlN })_{N}$ MULTILAYER FILMS WITH A TRANSITION LAYER}

In the stacking, between each nanolayer of TiAl and TiAlN, a transition layer was added (Figure 3.b.). For this transition layer, an elastoplastic metal/ceramic medium behaviour was considered. Each elastoplastic parameter of the transition layer was assumed to be an average (mixture law 50/50) of the parameters of the TiAl and TiAlN nanolayers. The thickness of this transition layer was optimised by minimising the gap between experimental and simulated data as previously described.

Result of the simulated (P-h) curve with a transition layer is plotted on Figure 5.a and show a good agreement between experimental and simulated curves for the lowest period. For $\Lambda=10 \mathrm{~nm}$, the simulated thickness of the transition layer, $\mathrm{ep}_{\mathrm{Tr}}=2.12 \mathrm{~nm}$, is close to the one of TiAl and TiAlN layers. This result is in very good agreement with the thickness of interfaces observed between TiAl and TiAlN layers evaluated by Electron Energy-Loss Spectroscopy (Figure 5 b).

\section{Conclusion}

The elastoplastic properties of $\mathrm{Ti}_{0.54} \mathrm{Al}_{0.46}$ metal and $\mathrm{Ti}_{0.54} \mathrm{Al}_{0.46} \mathrm{~N}$ ceramic monolayers were identified by comparison of Berkovich nanoindentation experimental data and 2D axisymmetric finite element simulations. The mechanical properties of the monolayers were used in a multilayer model to forecast the mechanical properties of $\left(\mathrm{Ti}_{0.54} \mathrm{Al}_{0.46} / \mathrm{Ti}_{0.54} \mathrm{Al}_{0.46} \mathrm{~N}\right)_{\mathrm{n}}$ multilayer coatings, with various periods, designed by RF magnetron sputtering using RGPP technique. The multilayer model was considered, in first hypothesis, as a stacking of TiAlN and TiAl nanolayers with equal thickness and perfect interface. That model allows to reproduce the indentation curves of the highest
$50 \mathrm{~nm}$ period films. To fit the experimental indentation curve at the lowest periods, a transition layer was introduced in the model between each metal/ceramic interface. Using an optimisation process of the model, this transition layer was found to be $2.12 \mathrm{~nm}$ thick for the lowest period $\Lambda=10 \mathrm{~nm}$, which is in good agreement with the results of Electron Energy-Loss Spectroscopy experiments.

\section{ACKNOWLEDGEMENTS}

This work was performed in the frame of the research project RESEM 2019, managed by the Institut de Recherche Technologique Matériaux Métallurgie Procédés (IRT M2P) and financially supported by the French program Plan d'Investissement d'Avenir (PIA).

\section{REFERENCES}

[1] M.-J. Pac, Y. Pinot, S. Giljean, et al. Investigation of Ti0.54Al0.46/Ti0.54Al0.46N multilayer films deposited by reactive gas pulsing process by nano-indentation and electron energy-loss spectroscopy. Thin Solid Films 634:96 - 106, 2017. DOI:10.1016/j.tsf.2017.05.015.

[2] W. Münz. Titanium aluminium nitride films: A new alternative to tin coatings. Journal of Vacuum Science 86 Technology A 4:2717-2725, 1986. DOI:10.1116/1.573713.

[3] S. PalDey, S. Deevi. Single layer and multilayer wear resistant coatings of $(\mathrm{Ti}, \mathrm{Al}) \mathrm{N}$ : a review. Materials Science and Engineering: A 342(1):58 - 79, 2003. DOI:10.1016/S0921-5093(02)00259-9

[4] M.-J. Pac, S. Giljean, C. Rousselot, et al. Microstructural and elasto-plastic material parameters identification by inverse finite elements method of Ti $(1-\mathrm{x})$ AlxN $(0<\mathrm{x}<1)$ sputtered thin films from berkovich nano-indentation experiments. Thin Solid Films 569:81 - 92, 2014. DOI:10.1016/j.tsf.2014.07.037.

[5] N. Martin, J. Lintymer, F. Sthal, et al. Procédé de pulvérisation réactive à signal de commande cyclique et dispositif correspondant 2008.

[6] K. Levenberg. A method for the solution of certain non-linear problems in least squares. Quarterly of Applied Mathematics 2(2):164-168, 1944. 
[7] D. W. Marquardt. An algorithm for least-squares estimation of nonlinear parameters. Journal of the Society for Industrial and Applied Mathematics 11(2):431-441, 1963. DOI:10.1137/0111030

[8] F. Richard. MIC2M software: Modeling and identification of the mechanical behavior of materials, 2000 . 